\title{
Fitotoxicidade de Herbicidas a Diferentes Cultivares de MILHO-PIPOCA ${ }^{1}$
}

\author{
Phytotoxicity of Herbicides to Different Popcorn Cultivars
}

\author{
FREITAS, S.P. ${ }^{2}$, MOREIRA, J.G. ${ }^{3}$, FREITAS, I.L.J. ${ }^{4}$, FREITAS JÚNIOR, S.P. ${ }^{5}$, \\ AMARAL JÚNIOR, A.T. ${ }^{2}$ e SILVA, V.Q.R. ${ }^{4}$
}

\begin{abstract}
RESUMO - Com o objetivo de avaliar a seletividade de diferentes herbicidas a dez genótipos de milho-pipoca em Campos dos Goytacazes-RJ, realizou-se a aplicação de herbicidas em dosagem máxima recomendada (atrazine + S-metolachlor + extravon - 1,665 + $1,035 \mathrm{~kg} \mathrm{ha}^{-1}$ i.a. $+0,1 \%$; foramsulfuron + iodosulfuron + hoefix $-45,0+3,0 \mathrm{~g} \mathrm{ha}^{-1}$ i.a. $+0,5 \%$; mesotrione + óleo mineral $-192 \mathrm{~g} \mathrm{ha}^{-1}+0,5 \%$; tembotrione + óleo mineral $-240 \mathrm{~mL} \mathrm{ha}^{-1}+$ $0,5 \%)$ e um tratamento sem aplicação de herbicida, em esquema fatorial $5 \times 10 \times 5.0$ experimento foi conduzido no delineamento em blocos casualizados, com quatro repetições. Aos 2, 4, 8, 12 e 20 dias após aplicação (DAA) foram avaliados sintomas visuais de fitotoxicidade. Aos 36 DAA foram avaliados a altura das plantas e o diâmetro do caule, e aos 39 DAA, a área foliar e a massa seca da parte aérea. Entre os tratamentos, os mais seletivos às variedades testadas foram os herbicidas atrazine + S-metolachlor, aplicados em pré-emergência; no entanto, atrazine + S-metolachlor, mesotrione e foramsulfuron + iodosulfuron, aplicados em pós-emergência, causaram elevados niveis de fitotoxicidade às plantas de milho-pipoca, sendo as variedades Beija-Flor, Pr-023, SE-013, Angela, PA-038 e UFV extremamente sensiveis a esses produtos.
\end{abstract}

Palavras-chave: seletividade, manejo de herbicida, Zea mays.

ABSTRACT - Aiming to evaluate the selectivity of different herbicides to 10 genotypes of popcorn in Campos dos Goytacazes-RJ, herbicides were applied at the recommended maximum dosage (atrazine + S-metolachlor + extravon $1.665+1.035 \mathrm{~kg} \mathrm{ha}^{-1}$ i.a. $+0.1 \%$; foramsulfuron + iodosulfuron + hoefix $45.0+3.0 \mathrm{~g} \mathrm{ha}^{-1}$ i.a. $+0.5 \%$; mesotrione + mineral oil $192 \mathrm{~g} \mathrm{ha}{ }^{-1}+0.5 \%$; tembotrione + mineral

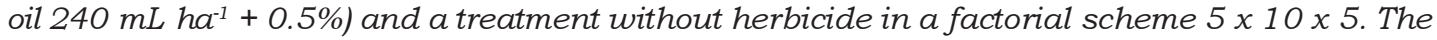
experiment was conducted in a randomized block design with four replications. Visual phytotoxicity symptoms were evaluated at 2, 4, 8, 12 and 20 days after application (DAA). Plant height and stem diameter were evaluated at 36 DAA, and leaf area and dry mass of the aerial part at $39 D A A$. The treatments found to be the most selective to the tested varieties were atrazine $+S$-metolachlor, applied in pre-emergence; however, atrazine + S-metolachlor, mesotrione and foramsulfuron + iodosulfuron, applied in post-emergence, caused high levels of phytotoxicity to the popcorn plants, with the varieties Beija-Flor, Pr-023, SE-013, Angela, PA-O38 and UFV being extremely sensitive to these products.

Keywords: selectivity, herbicide management, Zea mays.

\section{INTRODUÇÃO}

O milho-pipoca é alimento muito apreciado no Brasil; atualmente, em consequência do aprimoramento e da popularização de máquinas elétricas e fornos de micro-ondas para o pipocamento do milho, constata-se aumento crescente na produção e no consumo (Daros et al., 2004). Segundo Santos et al. (2007), o cultivo do milho-pipoca por meio do

1 Recebido para publicação em 31.10.2008 e na forma revisada em 11.12.2009

2 Professor associado da Universidade Estadual do Norte Fluminense Darcy Ribeiro - UENF, Campos dos Goytacazes-RJ, <silverio@uenf.br>; ${ }^{3}$ Engenheiro-Agrônomo, UENF; ${ }^{4}$ Pós-Graduando em Produção Vegetal, UENF; ${ }^{5}$ Doutor em Genética e Melhoramento de Plantas, UENF. 
sistema integrado entre empresas empacotadoras e produtores tem crescido no País. As empacotadoras selecionam a região onde querem produzir, fornecem as sementes e a tecnologia, favorecendo o aumento de produtividade e a redução nos custos de produção. Ainda segundo esses autores, na safra de 2006, os melhores produtores colheram, em média, 50 a 60 sacas por hectare e o custo de produção variou de $R \$ 800,00$ a $R \$ 1.200,00$ ha $^{-1}$, em função da tecnologia utilizada. As empresas empacotadoras pagaram aos cooperados cerca de $R \$ 45,00$ por saca.

O seu cultivo pode ser incentivado no Brasil, como alternativa à diversificação das atividades agrícolas, visto que possui alto valor econômico por área e pode-se agregar valor ao produto final. A adoção de variedades melhoradas, adubações e manejo integrado de pragas, doenças e plantas daninhas é necessária para se obter boa produtividade.

O controle químico de plantas daninhas tem se destacado pela eficiência, pela rapidez e pelo baixo custo; contudo, a eficácia dos herbicidas é variável entre si e dependente das condições ambientais, da época de aplicação e da espécie daninha a ser controlada (Jakelaitis et al., 2005). Devido à falta de informação sobre herbicidas seletivos para a cultura do milhopipoca, muitos agricultores utilizam, indevidamente, alguns produtos registrados para milho comum, colocando em risco o cultivo de milhopipoca. No entanto, alguns trabalhos estão sendo realizados para suprir a falta de informações sobre essa importante cultura. Silva et al. (2005) avaliaram o efeito da mistura de herbicidas nicosulfuron + atrazine e o inseticida chlorpirifos sobre o cultivar de milho-pipoca UFVM2, as plantas daninhas e a lagarta-docartucho. Esses autores não observaram interação significativa entre doses de nicosulfuron e do chlorpirifos no controle da lagartado-cartucho, e a mistura no tanque formada pelo chlorpirifos com os herbicidas promoveu intoxicação às plantas de milho-pipoca, principalmente nas maiores doses do nicosulfuron (20, 30 e $\left.40 \mathrm{~g} \mathrm{ha}^{-1}\right)$. O chlorpirifos não interferiu na eficiência de controle de plantas daninhas pelos herbicidas nem no estande final, no número de espigas por planta, no rendimento e na capacidade de expansão dos grãos de milho-pipoca.
Trabalhando com milho-pipoca, Jakelaitis et al. (2005) avaliaram a eficiência de controle de plantas daninhas, a tolerância e a produção na variedade UFVM2 aos herbicidas atrazine $\left(1.500 \mathrm{~g} \mathrm{ha}^{-1}\right)$, foramsulfuron + iodosulfuron methyl sodium + atrazine $(15+1+1.500 \mathrm{e}$ $30+2+1.500 \mathrm{~g} \mathrm{ha}^{-1}$ ), foramsulfuron + iodosulfuron methyl sodium $(15+1,30+2,45+3 \mathrm{e}$ $\left.60+4 \mathrm{~g} \mathrm{ha}^{-1}\right)$, nicosulfuron + atrazine $(8+1.500$ e $\left.16+1.500 \mathrm{~g} \mathrm{ha}^{-1}\right)$ e nicosulfuron $\left(16 \mathrm{~g} \mathrm{ha}^{-1}\right)$, concluindo que os herbicidas foramsulfuron + iodosulfuron methyl sodium, em todas as doses testadas, proporcionaram maiores indices de toxicidade à cultura, aos 7, 14 e 28 dias após a aplicação dos tratamentos (DAA). Todas as combinações de herbicidas proporcionaram controle acima de $90 \%$ para $B$. plantaginea e B. decumbens, aos 28 DAA. O nicosulfuron aplicado isoladamente proporcionou controle de apenas $80 \%$, e o atrazine não controlou essas espécies daninhas. Para Ipomoea spp., nenhum tratamento herbicida proporcionou controle equivalente ao da testemunha capinada. A competição das plantas daninhas resultou em menores teores de clorofila total, N, P e K no tecido foliar das plantas de milhopipoca e menor rendimento de grãos. Quanto ao cultivar UFVM2, as sulfonilureias isoladas ou associadas ao atrazine não alteraram o estado nutricional da cultura, o rendimento e a capacidade de expansão dos grãos de milho-pipoca.

Johnson et al. (2002) encontraram niveis de injúrias em plantas de milho pela aplicação do mesotrione em pós-emergência variando de 0 a 15\%, dependendo da época de aplicação e da dose utilizada. Em milho-doce, Sullivan \& Bouw (1997) verificaram que plantas tratadas com nicosulfuron na dose de $50 \mathrm{~g} \mathrm{ha}^{-1}$, aplicado nos estádios de cinco e sete folhas expandidas, apresentaram diversos sintomas de injúria, enquanto aquelas tratadas com $25 \mathrm{~g} \mathrm{ha}^{-1}$, nos mesmos estádios, foram pouco afetadas. Para o cultivar de milho-pipoca IAC 112, Sawazaki (2006) não observou redução no rendimento da cultura quando esta foi manejada com nicosulfuron na dose de $24 \mathrm{~g} \mathrm{ha}^{-1}$, em mistura com atrazine. Os tratamentos herbicidas foramsulfuron + iodosulfuron provocaram sintomas de fitotoxicidade inicial caracterizados por um leve amarelecimento das folhas da cultura. Nas avaliações subsequentes, os sintomas foram desaparecendo gradativamente e, aos 21 DAA, não mais se constatou 
nenhuma injúria. Cabe salientar que essas injúrias não provocaram qualquer efeito na produção da cultura.

Cavalieri et al. (2008) avaliaram a tolerância de híbridos de milho ao herbicida nicosulfuron e relacionaram estudos de seletividade desse herbicida conduzidos em casa de vegetação com estudos desenvolvidos em campo. Em casa de vegetação, após aplicação do herbicida, avaliou-se a massa seca da parte aérea das plantas. Em campo, foram avaliados o crescimento e a produtividade dos híbridos após aplicação do herbicida. Por meio dos resultados obtidos em casa de vegetação, foi possivel agrupar os híbridos em diferentes níveis de tolerância ao herbicida. Com relação à produtividade em campo, o híbrido B 761 apresentou redução significativa $(17,4 \%)$ na dose de $60 \mathrm{~g} \mathrm{ha}^{-1}$ do nicosulfuron.

Objetivou-se, neste trabalho, avaliar a seletividade de diferentes herbicidas registrados para milho comum, em genótipos de milho-pipoca.

\section{MATERIAL E MÉTODOS}

Dez variedades de milho-pipoca (UNB2UC3, Beija-Flor, UFV-Viçosa, Amarelo, PR-023 Maringá, Viçosa-Maringá, PA-038 Maringá, Branco, SE-013 Maringá e Angela-Embrapa) foram utilizadas em experimento implementado em sacolas plásticas mantidas em campo na Unidade de Apoio à Pesquisa (UAP) da Universidade Estadual do Norte Fluminense Darcy Ribeiro (UENF), em Campos dos Goytacazes-RJ, no delineamento em blocos casualizados com quatro repetições, em esquema fatorial $5 \times 10 \times 5$, para avaliar a reação a fitotoxicidade a quatro herbicidas, em cinco épocas diferentes: 2, 4, 8, 12 e 20 dias após a aplicação (DAA). Foram empregados os herbicidas mesotrione, foramsulfuron + iodosulfuron, atrazine + S-metolachlor, tembotrione, em pós-emergência; atrazine + S-metolachlor, em pré-emergência; e ausência de herbicidas (testemunha).

Foram avaliadas as características altura da planta (ALT), diâmetro do caule (DIAM), área foliar (AF) e massa seca da parte aérea (BSPA); na análise estatística, foram considerados os fatores e as variedades de milho-pipoca por cinco tipos de manejos com os diferentes herbicidas. Cada unidade experimental foi composta por uma sacola plástica com 12 litros de substrato (solo:areia; $2: 1 \mathrm{v}: \mathrm{v}$ ), contendo duas plantas de milho-pipoca por sacola, sendo as plantas cultivadas a céu aberto. Elas foram adubadas com $50 \mathrm{~g}$ por vaso da formulação 8-28-16 de $\mathrm{N}-\mathrm{P}_{2} \mathrm{O}_{5}-\mathrm{K}_{2} \mathrm{O}$, de acordo com a análise de solo.

Logo após a semeadura do milho-pipoca, foi realizada a irrigação até a capacidade de campo. O herbicida atrazine $+\mathrm{S}$-metolachlor foi aplicado no dia seguinte à semeadura, na dose de $1,665+1,035 \mathrm{~kg} \mathrm{ha}^{-1}$ i.a. A pulverização foi feita no início da manhã, com umidade relativa do ar (UR) de $70 \%$, temperatura de $25{ }^{\circ} \mathrm{C}$ e velocidade do vento em torno de $4 \mathrm{~km} \mathrm{ha}^{-1}$. A pulverização em pós-emergência foi realizada quando as plantas estavam no estádio 1 (quatro folhas expandidas), com os seguintes herbicidas e doses: atrazine + S-metolachlor + extravon - 1,665 + $1,035 \mathrm{~kg} \mathrm{ha}^{-1}$ i.a. $+0,1 \%$; foramsulfuron + iodosulfuron + hoefix $-45,0+3,0 \mathrm{~g} \mathrm{ha}^{-1}$ i.a. + $0,5 \%$; mesotrione + óleo mineral - $192 \mathrm{~g} \mathrm{ha}^{-1}+$ $0,5 \%$; e tembotrione + óleo mineral $240 \mathrm{~mL} \mathrm{ha} \mathrm{h}^{-1}+0,5 \%$. Em todas as aplicações foi utilizado pulverizador costal, pressurizado a $\mathrm{CO}_{2}$, com pressão constante de $3,5 \mathrm{kgf} \mathrm{cm}^{-2}$, e equipado com bico XR 8003 EVS, calibrado para aplicar o equivalente a $300 \mathrm{~L} \mathrm{ha}^{-1}$ de calda. Após a aplicação de cada produto, o pulverizador era lavado com água e detergente, a fim de evitar misturas dos herbicidas nos tratamentos posteriores. As plantas foram irrigadas diariamente, e o teor de umidade nas sacolas foi mantido próximo à capacidade de campo.

A avaliação de fitotoxicidade foi realizada aos 2, 4, 8, 12 e 20 dias após a aplicação (DAA) dos herbicidas, sendo determinada por comparação visual, em que foram atribuídas notas de zero (0) (ausência de fitotoxicidade) a cem (100\%) (morte das plantas), de acordo com Frans (1972).

Aos 36 DAA dos herbicidas, foram avaliadas as seguintes variáveis: altura das plantas (ALT) medida a partir da base da planta - rente ao solo - até a bainha da última folha completamente desenvolvida - bainha completamente formada), por meio de uma trena milimetrada; e diâmetro do caule (DIAM) (medido a $5 \mathrm{~cm}$ de altura em relação ao solo, utilizando um paquímetro). 
Aos 39 DAA, foram cortadas, ao nivel do solo, as duas plantas do vaso em todas as parcelas, para obtenção do índice de área foliar (AF) e massa seca da parte aérea (BSPA). O índice de área foliar (AF) foi estimado, no laboratório, por meio de um medidor de área foliar de bancada LI-3100 (LICOR, Nebraska, USA). Para estimar a massa seca da parte aérea (BSPA), esta foi acondicionada em uma estufa com ventilação forçada, em temperatura de $70{ }^{\circ} \mathrm{C}$, até atingir peso constante.

Os dados obtidos foram analisados estatisticamente por meio da aplicação do teste $\mathrm{F}$ sobre a análise de variância, seguida do teste de Scott-Knott (Steel \& Torrie, 1980), para agrupamento de médias das interações, ambos a $5 \%$ de probabilidade.

\section{RESULTADOS E DISCUSSÃO}

De acordo com a Tabela 1, nota-se que as características altura da planta (ALT), diâmetro do caule (DIAM), massa seca da parte aérea (BSPA) e área foliar (AF) apresentaram diferenças significativas na fonte de variação manejo (MAN), bem como interação significativa entre herbicida e variedades utilizadas no experimento para todas as características (Tabela 1), indicando que as variedades e os herbicidas tiveram desempenho diferente.

Avaliando a variável fitotoxicidade, nota-se que os quadrados médios das fontes de variação manejos (MAN) e dias após a aplicação do herbicida (TEMP) foram significativos (Tabela 2), o que é um indicativo de que os herbicidas e os tempos utilizados no presente trabalho apresentaram diferenças. Em consonância com esses resultados, nota-se que a interação MAN $x$ TEMP x VAR (Tabela 2) apresentou-se significativa, o que confirma que os tratamentos utilizados tiveram respostas diferentes.

Quanto à característica fitotoxicidade, ocorreram diferenças significativas entre os diferentes dias após a aplicação do herbicida (DAA), aos 2, 4, 8, 12 e 20 DAA. Isso evidencia a redução dos sintomas no decorrer das avaliações. Aos 20 DAA, não foram perceptiveis os sintomas visuais de intoxicação nas folhas, exceto no tratamento em que foi aplicado o herbicida atrazine + S-metolachlor (pós), que

Tabela 2 - Valores e significância dos quadrados médios (QM) e coeficientes de variação com base nas médias dos tratamentos da variável fitotoxicidade. Campos dos Goytacazes-RJ, 2006

\begin{tabular}{|l|r|c|}
\hline \multirow{2}{*}{ FV } & \multirow{2}{*}{ GL } & Quadrado Médio \\
\cline { 3 - 3 } & & Fitotoxicidade \\
\hline (B/V)/T & 150 & 289,38 \\
\hline Manejo (MAN) & 4 & $247708,03^{*}$ \\
\hline Tempo (TEMP) & 4 & $15519,09^{*}$ \\
\hline Variedades (VAR) & 9 & $4856,01^{\mathrm{ns}}$ \\
\hline MAN x TEMP & 20 & $2690,98^{*}$ \\
\hline MAN x VAR & 45 & $4320,46^{*}$ \\
\hline TEMP x VAR & 36 & $492,08^{\mathrm{ns}}$ \\
\hline MAN x TEMP x VAR & 180 & $380,82^{*}$ \\
\hline Resíduo & 750 & 178,64 \\
\hline Média & & 30,76 \\
\hline CV(\%) & & 43,45 \\
\hline
\end{tabular}

is não significativo a $5 \%$ de probabilidade pelo teste $\mathrm{F}$; * significativo a $5 \%$ de probabilidade pelo teste $\mathrm{F}$.

Tabela 1 - Valores e significância dos quadrados médios e coeficientes de variação referentes ao uso de quatro herbicidas em dez variedades de milho-pipoca. Campos dos Goytacazes-RJ, 2006

\begin{tabular}{|l|c|c|c|c|c|}
\hline \multirow{2}{*}{ FV } & \multirow{2}{*}{ GL } & \multicolumn{4}{|c|}{${\text { Quadrado } \text { Médio }^{\text {1/ }}}$} \\
\cline { 3 - 6 } & & ALT & DIAM & BSPA & AF \\
\hline Blocos & 3 & 263,40 & 50,77 & 4256,06 & 4672241,28 \\
\hline Manejo (MAN) & 4 & $45639,30^{*}$ & $2123,18^{*}$ & $50949,07^{*}$ & $248148543,02^{*}$ \\
\hline Variedades (VAR) & 9 & $1570,87^{\text {ns }}$ & $80,88^{\text {ns }}$ & $2781,10^{\text {ns }}$ & $11093206,05^{\text {ns }}$ \\
\hline MAN x VAR & 45 & $1304,75^{*}$ & $52,26^{*}$ & $1566,65^{*}$ & $7192676,19^{*}$ \\
\hline Resíduo & 177 & 347,54 & 24,41 & 874,03 & 4213170,31 \\
\hline Média & & 62,71 & 12,96 & 58,95 & 4150,07 \\
\hline CV(\%) & & 29,73 & 38,11 & 50,15 & 49,46 \\
\hline
\end{tabular}

1/ ALT = altura da planta; DIAM = diâmetro do caule; $\mathrm{BSPA}=$ massa seca da parte aérea; $\mathrm{AF}=$ área foliar.

${ }^{\text {ns }}$ não significativo a $5 \%$ de probabilidade pelo teste $\mathrm{F}$; ${ }^{*}$ significativo a $5 \%$ de probabilidade pelo teste $\mathrm{F}$. 
causou morte em quase todas as variedades; somente a variedade Viçosa-Maringá conseguiu se recuperar aos 20 DAA. Já o herbicida mesotrione proporcionou danos reversiveis nas diferentes variedades; PA-038, UFV e Beija-Flor tiveram danos irreparáveis. Portanto, o herbicida atrazine + S-metolachlor, aplicado em pós-emergência, foi o que mais causou injúrias às plantas de milho-pipoca quando comparado com a testemunha, seguido dos herbicidas mesotrione e foramsulfuron + iodosulfuron (Tabela 3). No entanto, os herbicidas atrazine + S-metolachlor, aplicados em pré-emergência, e tembotrione foram os mais seletivos quando comparados à testemunha.

Na Tabela 3, pode-se constatar que as variedades UNB2U-C3, Beija-Flor, UFV, Amarelo, PR-023, PA-038, Branco, SE-013 e Angela são altamente sensiveis ao herbicida atrazine + S-metolachlor (pós), todos com 100\% de fitotoxicidade.

As variedades de milho-pipoca que receberam o manejo do herbicida mesotrione, aplicado em pós-emergência, apresentaram, inicialmente, clorose, a qual se seguiu de acentuado albinismo nas folhas novas - sintoma intrínseco aos inibidores de pigmentos, grupo ao qual o mesotrione pertence. Esses sintomas de toxicidade se agravaram ao longo do tempo nas variedades PA-038, UFV e Beija-Flor, causandolhes a morte (100\% de fitotoxicidade) aos 2,12 e 20 DAA, respectivamente (Tabela 3).

Por sua vez, para as variedades UNB2UC3, Amarelo, UFV, PR-023, Branco, SE-013, Angela e Viçosa-Maringá, apesar dos severos sintomas em suas folhas nos primeiros dias, ocorreu acentuada redução deles durante as avaliações subsequentes até a recuperação dos sintomas visuais, não diferindo estatisticamente da testemunha, manejada sem aplicação de herbicida aos 20 DAA (Tabela 3).

Experimentos realizados na Embrapa Milho e Sorgo (Embrapa, 2005) para testar a seletividade do mesotrione a seis cultivares de milho comum revelaram que o maior indice de fitotoxicidade foi no cultivar BRS 2020, aos 14 dias após a aplicação. Nesse caso, a maior fitotoxicidade se deu já aos 2 DAA do herbicida, sendo letal às variedades SE-013, Beija-Flor, UFV e PR-023. No presente trabalho com milhopipoca, ocorreu estresse causado pela ação do herbicida, com base no que foi observado nas variedades UNB2U-C3, Amarelo UFV, PR-023, Branco, SE-013 e Angela, que tiveram acentuada redução na altura em relação à testemunha. Essa redução foi de magnitude de 15,2 a 47,3\% nas variedades Angela, Amarelo e UFV (Tabela 4).

Quanto às variáveis diâmetro do caule (DIAM), massa da parte aérea (BSPA) e área foliar (AF), as variedades UNB2U-C3, Branco, UFV e SE-013 expressaram médias que não diferiram estatisticamente pelo teste de ScottKnott, mostrando tendência decrescente nos seus valores (Tabela 4).

O tratamento com o herbicida foramsulfuron + iodosulfuron, aplicado em pós-emergência, causou toxicidade em todas as plantas, mas em menor intensidade que os manejos supracitados. Aos 2 DAA, todas as variedades apresentaram pelo menos algum efeito tóxico, seja na descoloração das folhas ou no retardo do crescimento. Esses sintomas foram desaparecendo no decorrer das avaliações (Tabela 3). Aos 20 DAA, entre as variedades tratadas com o herbicida, pode-se considerar que apenas a PA-038 (50\% de fitotoxicidade) não conseguiu recuperar-se dos danos causados por ele, sendo a variedade mais sensivel ao foramsulfuron + iodosulfuron (Tabela 3). A altura das plantas de PA-038 foi 72,3\% menor; o diâmetro, 63,3\% menor; a massa seca da parte aérea, 83\% menor; e a área foliar, 89,2\% menor que sua testemunha, comprometendo o crescimento da planta no final do experimento (Tabela 4). As variedades Beija-Flor, UFV, Amarelo e ViçosaMaringá não diferiram estatisticamente quanto à variável altura de planta, mas nas demais características avaliadas a variedade Viçosa-Maringá se destacou. Contudo, o diâmetro do caule, a massa seca e a área foliar para a variedade Viçosa-Maringá não diferiram estatisticamente da testemunha, expressando boa tolerância ao foramsulfuron + iodosulfuron (Tabela 4).

No entanto, a utilização de herbicidas inibidores da acetolactato sintase, como o caso do foramsulfuron + iodosulfuron, pode causar toxidez diferencial em híbridos e variedades de milho (Damião Filho et al., 1996), devido à diferente capacidade destes para metabolizá-los a compostos não tóxicos (Brown, 1990; Bunting et al., 2004). 
Tabela 3 - Valores médios de fitotoxicidade resultantes da interação entre as diferentes variedades e manejos realizados, aos $2,4,8,12$ e 20 dias após a aplicação (DAA). Campos dos Goytacazes-RJ, 2006

\begin{tabular}{|c|c|c|c|c|c|}
\hline \multirow{2}{*}{ Tratamento } & \multicolumn{5}{|c|}{ Média de fitotoxicidade } \\
\hline & $2 \mathrm{DAA}$ & 4 DAA & 8 DAA & $12 \mathrm{DAA}$ & $20 \mathrm{DAA}$ \\
\hline Beija-Flor $\mathrm{x}$ atrazine + S-metolachlor (pós) & $100,00 \mathrm{a}$ & $100,00 \mathrm{a}$ & $100,00 \mathrm{a}$ & $100,00 \mathrm{a}$ & $100,00 \mathrm{a}$ \\
\hline PR-023 $\mathrm{x}$ atrazine + S-metolachlor (pós) & $100,00 \mathrm{a}$ & $100,00 \mathrm{a}$ & $100,00 \mathrm{a}$ & $100,00 \mathrm{a}$ & $100,00 \mathrm{a}$ \\
\hline SE-013 $\times$ atrazine + S-metolachlor (pós) & $100,00 \mathrm{a}$ & $100,00 \mathrm{a}$ & $100,00 \mathrm{a}$ & $100,00 \mathrm{a}$ & $100,00 \mathrm{a}$ \\
\hline Angela $\mathrm{x}$ atrazine + S-metolachlor (pós) & $98,75 \mathrm{a}$ & $100,00 \mathrm{a}$ & $100,00 \mathrm{a}$ & $100,00 \mathrm{a}$ & $100,00 \mathrm{a}$ \\
\hline Amarelo $\mathrm{x}$ atrazine + S-metolachlor (pós) & $95,00 \mathrm{a}$ & $80,00 \mathrm{~b}$ & 88,75 a & $100,00 \mathrm{a}$ & $100,00 \mathrm{a}$ \\
\hline PA-038 $\mathrm{x}$ atrazine + S-metolachlor (pós) & $95,00 \mathrm{a}$ & $100,00 \mathrm{a}$ & $100,00 \mathrm{a}$ & $100,00 \mathrm{a}$ & $100,00 \mathrm{a}$ \\
\hline Branco $\mathrm{x}$ atrazine + S-metolachlor (pós) & $95,00 \mathrm{a}$ & $95,00 \mathrm{a}$ & 97,50 a & $100,00 \mathrm{a}$ & $100,00 \mathrm{a}$ \\
\hline UNB $x$ atrazine + S-metolachlor (pós) & 93,75 a & $100,00 \mathrm{a}$ & $100,00 \mathrm{a}$ & $100,00 \mathrm{a}$ & $100,00 \mathrm{a}$ \\
\hline Amarelo x mesotrione & $90,00 \mathrm{a}$ & $80,00 \mathrm{~b}$ & $36,25 \mathrm{~d}$ & $10,00 \mathrm{e}$ & $0,00 \mathrm{c}$ \\
\hline PA-038 x mesotrione & 87,50 a & $67,50 \mathrm{c}$ & $61,25 \mathrm{c}$ & $75,00 \mathrm{~b}$ & $100,00 \mathrm{a}$ \\
\hline Branco x mesotrione & 86,25 a & $82,50 \mathrm{~b}$ & $46,25 \mathrm{~d}$ & $10,00 \mathrm{e}$ & $2,50 \mathrm{c}$ \\
\hline UFV x mesotrione & 86,25 a & $100,00 \mathrm{a}$ & $100,00 \mathrm{a}$ & $100,00 \mathrm{a}$ & $100,00 \mathrm{a}$ \\
\hline SE-013 x mesotrione & 83,75 a & $45,00 \mathrm{~d}$ & $45,00 \mathrm{~d}$ & $45,00 \mathrm{c}$ & $30,00 \mathrm{c}$ \\
\hline UNB x mesotrione & $81,25 \mathrm{a}$ & $82,50 \mathrm{~b}$ & $75,00 \mathrm{~b}$ & $56,25 \mathrm{~b}$ & $17,50 \mathrm{c}$ \\
\hline PR-023 x mesotrione & $78,75 \mathrm{a}$ & $30,00 \mathrm{~d}$ & $30,00 \mathrm{~d}$ & $21,25 \mathrm{~d}$ & $7,50 \mathrm{c}$ \\
\hline Angela x mesotrione & $78,75 \mathrm{a}$ & $70,00 \mathrm{c}$ & $46,25 \mathrm{~d}$ & $23,75 \mathrm{~d}$ & $7,50 \mathrm{c}$ \\
\hline Beija-Flor x mesotrione & $77,50 \mathrm{a}$ & $81,25 \mathrm{~b}$ & $78,75 \mathrm{~b}$ & $100,00 \mathrm{a}$ & $100,00 \mathrm{a}$ \\
\hline Viçosa-Maringá x mesotrione & $72,50 \mathrm{~b}$ & $40,00 \mathrm{~d}$ & $35,00 \mathrm{~d}$ & $15,00 \mathrm{~d}$ & $3,75 \mathrm{c}$ \\
\hline UFV $\mathrm{x}$ atrazine + S-metolachlor (pós) & $71,25 \mathrm{~b}$ & $70,00 \mathrm{c}$ & 87,50 a & $100,00 \mathrm{a}$ & $100,00 \mathrm{a}$ \\
\hline PA-038 x foramsulfuron + iodosulfuron & $70,00 \mathrm{~b}$ & $58,75 \mathrm{c}$ & $58,75 \mathrm{c}$ & $51,25 \mathrm{~b}$ & $50,00 \mathrm{~b}$ \\
\hline PR-023 x foramsulfuron + iodosulfuron & $62,50 \mathrm{~b}$ & $17,50 \mathrm{e}$ & $17,50 \mathrm{e}$ & $10,00 \mathrm{e}$ & $0,00 \mathrm{c}$ \\
\hline Viçosa-Maringá x atrazine + S-metolachlor (pós) & $53,75 \mathrm{~b}$ & $27,50 \mathrm{~d}$ & $17,50 \mathrm{e}$ & $6,25 \mathrm{e}$ & $0,00 \mathrm{c}$ \\
\hline Branco $\mathrm{x}$ foramsulfuron + iodosulfuron & $53,75 \mathrm{~b}$ & $40,00 \mathrm{~d}$ & $40,00 \mathrm{~d}$ & $21,25 \mathrm{~d}$ & $0,00 \mathrm{c}$ \\
\hline SE-013 $\times$ atrazine + S-metolachlor (pré) & $53,75 \mathrm{~b}$ & $16,25 \mathrm{e}$ & $16,25 \mathrm{e}$ & $0,00 \mathrm{e}$ & $0,00 \mathrm{c}$ \\
\hline UFV $x$ foramsulfuron + iodosulfuron & $52,50 \mathrm{~b}$ & $17,50 \mathrm{e}$ & $17,50 \mathrm{e}$ & $10,00 \mathrm{e}$ & $0,00 \mathrm{c}$ \\
\hline PA-038 $x$ tembotrione & $50,00 \mathrm{~b}$ & $13,75 \mathrm{e}$ & $2,50 \mathrm{e}$ & $0,00 \mathrm{e}$ & $0,00 \mathrm{c}$ \\
\hline Angela $\mathrm{x}$ foramsulfuron + iodosulfuron & $50,00 \mathrm{~b}$ & $52,50 \mathrm{c}$ & $52,50 \mathrm{c}$ & $20,00 \mathrm{~d}$ & $0,00 \mathrm{c}$ \\
\hline Beija-Flor $\mathrm{x}$ foramsulfuron + iodosulfuron & $42,50 \mathrm{c}$ & $12,50 \mathrm{e}$ & $12,50 \mathrm{e}$ & $3,75 \mathrm{e}$ & $0,00 \mathrm{c}$ \\
\hline Angela $\mathrm{x}$ atrazine + S-metolachlor (pré) & $38,75 \mathrm{c}$ & $38,75 \mathrm{~d}$ & $15,00 \mathrm{e}$ & $0,00 \mathrm{e}$ & $0,00 \mathrm{c}$ \\
\hline SE-013 $\times$ foramsulfuron + iodosulfuron & $37,50 \mathrm{c}$ & $30,00 \mathrm{~d}$ & $30,00 \mathrm{~d}$ & $15,00 \mathrm{~d}$ & $0,00 \mathrm{c}$ \\
\hline Viçosa-Maringá $\mathrm{x}$ atrazine + S-metolachlor (pré) & $35,00 \mathrm{c}$ & $28,75 \mathrm{~d}$ & $28,75 \mathrm{~d}$ & $27,50 \mathrm{~d}$ & $0,00 \mathrm{c}$ \\
\hline Amarelo x Foramsulfuron + iodosulfuron & $22,50 \mathrm{~d}$ & $18,75 \mathrm{e}$ & $18,75 \mathrm{e}$ & $7,50 \mathrm{e}$ & $0,00 \mathrm{c}$ \\
\hline Viçosa-Maringá x foramsulfuron + iodosulfuron & $22,50 \mathrm{~d}$ & $23,75 \mathrm{~d}$ & $23,75 \mathrm{~d}$ & $8,75 \mathrm{e}$ & $0,00 \mathrm{c}$ \\
\hline UNB $x$ foramsulfuron + iodosulfuron & $20,00 \mathrm{~d}$ & $23,75 \mathrm{~d}$ & $23,75 \mathrm{~d}$ & $10,00 \mathrm{e}$ & $0,00 \mathrm{c}$ \\
\hline Viçosa-Maringá $\mathrm{x}$ tembotrione & $18,75 \mathrm{~d}$ & $6,25 \mathrm{e}$ & $2,50 \mathrm{e}$ & $0,00 \mathrm{e}$ & $0,00 \mathrm{c}$ \\
\hline UNB $\mathrm{x}$ tembotrione & $15,00 \mathrm{e}$ & $3,75 \mathrm{e}$ & $0,00 \mathrm{e}$ & $0,00 \mathrm{e}$ & $0,00 \mathrm{c}$ \\
\hline Branco $\mathrm{x}$ tembotrione & $15,00 \mathrm{e}$ & $7,50 \mathrm{e}$ & $2,50 \mathrm{e}$ & $0,00 \mathrm{e}$ & $0,00 \mathrm{c}$ \\
\hline PR-023 x tembotrione & $13,75 \mathrm{e}$ & $6,25 \mathrm{e}$ & $1,25 \mathrm{e}$ & $0,00 \mathrm{e}$ & $0,00 \mathrm{c}$ \\
\hline Branco $\mathrm{x}$ atrazine + S-metolachlor (pré) & $12,50 \mathrm{e}$ & $13,75 \mathrm{e}$ & $13,75 \mathrm{e}$ & $0,00 \mathrm{e}$ & $0,00 \mathrm{c}$ \\
\hline PR-023 $\mathrm{x}$ atrazine + S-metolachlor (pré) & $11,25 \mathrm{e}$ & $3,75 \mathrm{e}$ & $3,75 \mathrm{e}$ & $0,00 \mathrm{e}$ & $0,00 \mathrm{c}$ \\
\hline PA-038 $\mathrm{x}$ atrazine + S-metolachlor (pré) & $11,25 \mathrm{e}$ & $7,50 \mathrm{e}$ & $7,50 \mathrm{e}$ & $0,00 \mathrm{e}$ & $0,00 \mathrm{c}$ \\
\hline SE-013 $\mathrm{x}$ aembotrione & $11,25 \mathrm{e}$ & $11,25 \mathrm{e}$ & $2,50 \mathrm{e}$ & $0,00 \mathrm{e}$ & $0,00 \mathrm{c}$ \\
\hline UFV $\mathrm{x}$ atrazine + S-metolachlor (pré) & $11,25 \mathrm{e}$ & $11,25 \mathrm{e}$ & $11,25 \mathrm{e}$ & $0,00 \mathrm{e}$ & $0,00 \mathrm{c}$ \\
\hline Beija-Flor $\mathrm{x}$ atrazine + S-metolachlor (pré) & $10,00 \mathrm{e}$ & $5,00 \mathrm{e}$ & $5,00 \mathrm{e}$ & $0,00 \mathrm{e}$ & $0,00 \mathrm{c}$ \\
\hline Amarelo $\mathrm{x}$ atrazine + S-metolachlor (pré) & $10,00 \mathrm{e}$ & $5,00 \mathrm{e}$ & $5,00 \mathrm{e}$ & $0,00 \mathrm{e}$ & $0,00 \mathrm{c}$ \\
\hline UNB $\mathrm{x}$ atrazine + S-metolachlor (pré) & $8,75 \mathrm{e}$ & $5,00 \mathrm{e}$ & 5,00 e & $0,00 \mathrm{e}$ & $0,00 \mathrm{c}$ \\
\hline Beija-Flor $\mathrm{x}$ tembotrione & $8,75 \mathrm{e}$ & $8,75 \mathrm{e}$ & $1,25 \mathrm{e}$ & $0,00 \mathrm{e}$ & $0,00 \mathrm{c}$ \\
\hline Amarelo $\mathrm{x}$ tembotrione & $7,50 \mathrm{e}$ & $7,50 \mathrm{e}$ & $1,25 \mathrm{e}$ & $0,00 \mathrm{e}$ & $0,00 \mathrm{c}$ \\
\hline UFV $\mathrm{x}$ tembotrione & $7,50 \mathrm{e}$ & $5,00 \mathrm{e}$ & $0,00 \mathrm{e}$ & $0,00 \mathrm{e}$ & $0,00 \mathrm{c}$ \\
\hline Angela $\mathrm{x}$ tembotrione & $7,50 \mathrm{e}$ & $3,75 \mathrm{e}$ & $0,00 \mathrm{e}$ & $0,00 \mathrm{e}$ & $0,00 \mathrm{c}$ \\
\hline
\end{tabular}

Médias seguidas de mesma letra, na mesma coluna, não diferem estatisticamente entre si pelo teste de Scott-Knott a 5\% de probabilidade 
Tabela 4 - Valores médios de altura da planta (ALT), diâmetro do caule (DIAM) aos 36 DAA e valores médios de massa seca da parte aérea (BSPA) e área foliar (AF) aos 39 DAA, resultantes da interação entre as diferentes variedades e manejos realizados. Campos dos Goytacazes-RJ, 2006

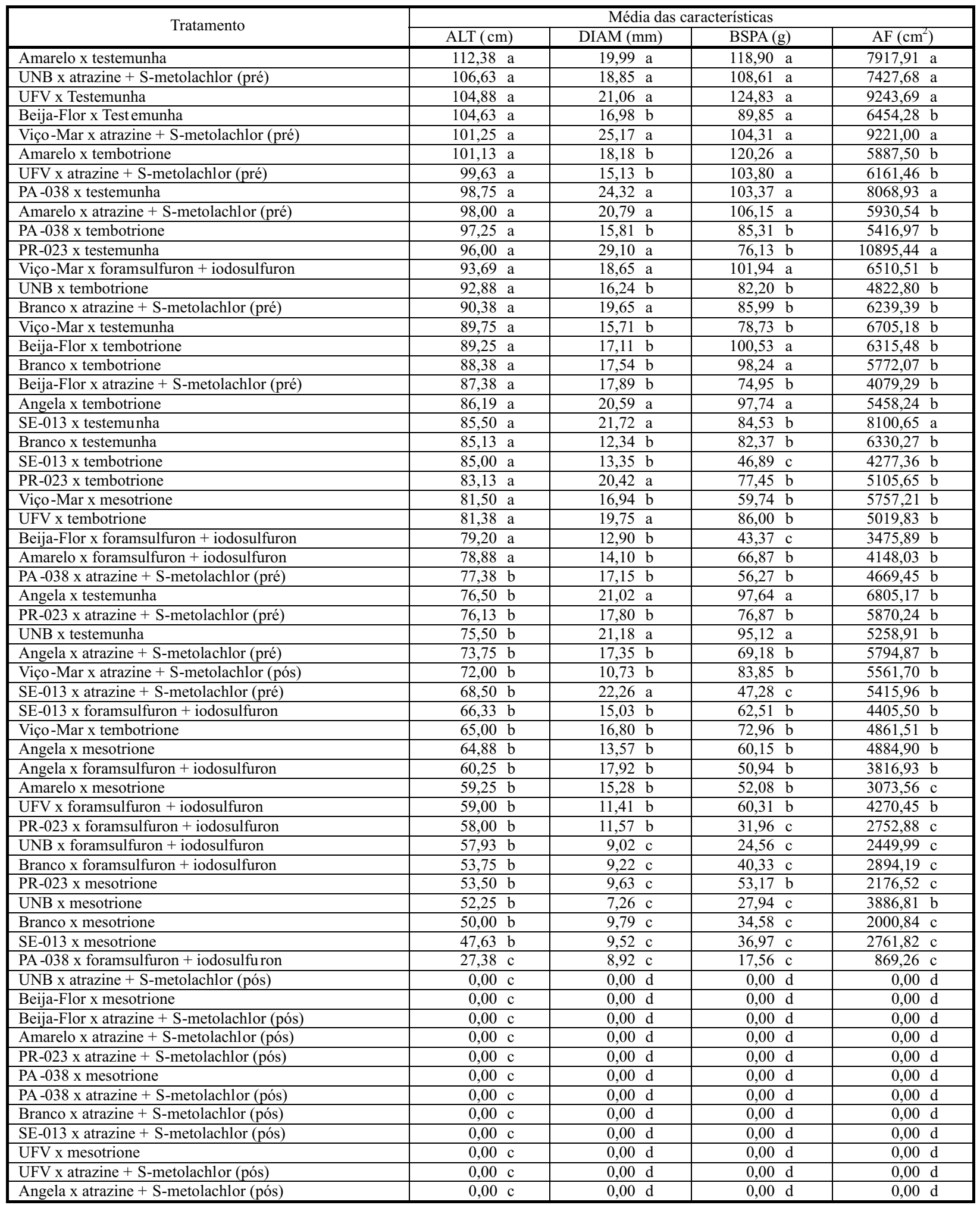

Médias seguidas de mesma letra, na mesma coluna, não diferem estatisticamente entre si pelo teste de Scott-Knott a 5\% de probabilidade. 
O manejo com o herbicida tembotrione, em pós-emergência, causou leve toxicidade às variedades testadas. Apenas a variedade PA-038 foi sensivel ao herbicida aos 2 DAA (50\% de fitotoxicidade), seguida de ViçosaMaringá $(18,75 \%)$. Ambas apresentaram rápida recuperação e, a partir do 8을 DAA, já não mostravam diferença significativa das médias da testemunha (Tabela 3).

Em relação às outras quatro variáveis (ALT, DIAM, BSPA e AF), a variedade que mais se destacou como tolerante ao tembotrione foi Angela, pois mostrou média inferior à da testemunha apenas em relação à área foliar. No entanto, as variedades UNB2U-C3, UFV, PR-023, Beija-Flor, Amarelo, PA-038, Branco e Viçosa-Maringá tiveram boa tolerância ao herbicida tembotrione (Tabela 4). Os resultados desta pesquisa corroboram os obtidos por Constantin et al. (2006), que obtiveram recuperação do milho comum a partir dos 17 DAA em todos os tratamentos, não ocorrendo mais qualquer sintoma de intoxicação, porém as variedades utilizadas no experimento demonstraram elevada capacidade de tolerar o herbicida, de maneira que aos 8 DAA já não havia sintomas visuais de intoxicação.

$\mathrm{O}$ manejo utilizando atrazine $+\mathrm{S}$-metolachlor em pré-emergência foi o que menos causou sintomas visuais de toxicidade às variedades de milho-pipoca testadas. Aos 2 DAA, as variedades SE-013, Angela e ViçosaMaringá apresentavam 53,75, 38,75 e 35\% de fitotoxicidade, respectivamente, enquanto as médias das outras variedades não diferiam significativamente da testemunha. No entanto, aos 12 DAA nenhum sintoma foi constatado em suas folhas. As variedades UNB2U-C3 e Viçosa-Maringá foram altamente tolerantes ao herbicida e não houve diferença significativa entres suas médias e a da testemunha para altura de planta, diâmetro do caule, massa seca da parte aérea e área foliar. Todavia, as demais variedades expressaram tolerância satisfatória ao herbicida, já que a altura, o diâmetro do caule, a massa seca e a área foliar não foram tão inferiores aos obtidos pela testemunha, apesar de diferirem estatisticamente.

Supõe-se que a maior seletividade do herbicida atrazine $+\mathrm{S}$-metolachlor, aplicado em pré-emergência, aos genótipos avaliados está relacionada à capacidade destes de metabolizar os princípios ativos que o compõem, transformando-os em metabólitos não tóxicos às plantas. Segundo López-Ovejero (2000), a evolução dos sintomas de fitotoxicidade até a aparente total recuperação das plantas ainda pode reservar reduções da produtividade final da cultura, já que o período de recuperação dos danos fitotóxicos pode reduzir o rendimento final. Os herbicidas atrazine $+\mathrm{S}$-metolachlor, em pré-emergência, e tembotrione foram seletivos às seguintes variedades: UNB2U-C3, Angela, UFV, PR-023, Beija-Flor, Amarelo, PA038, Branco e Viçosa-Maringá. Essas informações são valiosas tanto para agricultores quanto para pesquisadores envolvidos com a cultura, pois definem parâmetros técnicos referentes ao uso correto e eficiente dos herbicidas registrados para a cultura do milho-pipoca, evitando transtornos, perdas de produção no campo e redução da qualidade do produto final.

Os programas de seleção recorrente com milho-pipoca necessitam da incorporação de estudos sobre a seletividade a herbicidas, posto que materiais melhorados em fase de melhoramento tendem à maior sensibilidade à aplicação de herbicidas.

Os herbicidas menos seletivos às variedades testadas foram atrazine $+\mathrm{S}$-metolachlor, aplicado em pós-emergência, e mesotrione e foramsulfuron + iodosulfuron, ambos aplicados em pós-emergência.

\section{AGRADECIMENTOS}

À UENF, pelo suporte logístico na realização do experimento, e à FAPERJ, pelo apoio financeiro.

\section{LITERATURA CITADA}

BROWN, H. M. Mode of action, crop selectivity, and soil relations of the sulfonylurea herbicides. Pest. Sci., v. 29, p. $263-281,1990$

BUNTING, J. A.; SPRAGUE, C. L.; RIECHERS, D. E Physiological basis for tolerance of corn hybrids to foramsulfuron. Weed Sci., v. 52, p. 711-717, 2004.

CAVALIERI, S. D.et al. Tolerância de híbridos de milho ao herbicida nicosulfuron. Planta Daninha, v. 26, n. 1, p. $203-214,2008$ 
CONSTANTIN, J. et al. Seletividade e eficácia agronômica do novo herbicida tembotrione para a cultura do milho. In: CONGRESSO BRASILEIRO DA CIÊNCIA DAS PLANTAS DANINHAS, 25., 2006, Brasília. Anais... Brasília: 2006. v. 1.

DAMIÃO FILHO, C. F.; MÔRO, F. V.; TAVEIRA, L. R Respostas de híbridos de milho a nicosulfuron. Aspectos biológicos e da produção. Planta Daninha, v. 14, n. 1, p. 3-13, 1996.

DAROS, M. et al. Recurrent selection in inbred popcorn families. Sci. Agric., v. 61, n. 6, p. 609-614, 2004

EMPRESA BRASILEIRA DE PESQUISA AGROPECUÁRIA - EMBRAPA. Características do herbicida mesotrione na cultura do milho. Sete Lagoas: 2005. 5 p. (Circular Técnica, 52)

FRANS, R. E. Measuring plant response. In: WIKINSON, R. E. (Ed). Research methods in weed science. [S.1.]: Southern Weed Science Society, 1972. p. 28-41.

JAKELAITIS, A. et al. Controle de plantas daninhas na cultura do milho-pipoca com herbicidas aplicados em pósemergência. Planta Daninha, v. 23, n. 3, p. 509-516, 2005.
JOHNSON, B. C.; YOUNG, B. G.; MATTHEWS, J. L. Effect of post emergence application rate and timing of mesotrione on corn (Zea mays) response and weed control. Weed Technol., v. 16, p. 414-420, 2002.

LÓPEZ-OVEJERO, R.F. Desempenho da cultura de milho (Zea mays L.) submetida a diferentes herbicidas na ausência de plantas daninhas. 2000. 46 f. Dissertação (Mestrado em Fitotecnia) - Escola Superior de Agricultura "Luiz de Queiroz", Piracicaba, 2000.

SANTOS, F. S. et al. Predição de ganhos genéticos por índices de seleção na população de milho-pipoca UNB-2U sob seleção recorrente. Bragantia, v. 66, n. 3, p. 389-396, 2007.

SAWAZAKI, E. A cultura do milho-pipoca no Brasil. O agronômico, v. 53, n. 2. Disponível em: $<$ www.iac.sp.gov.br>. Acesso em: 2 set 2006

STEEL, R. G. D.; TORRIE, J. H. Principles and procedures of statistics: a biometrical approach. New York: McGraw-Hill Book Company, 1980. p. 633.

SULLIVAN, J. O.; BOUW, W. J. Sensitivity of processing sweet corn (Zea mays) cultivars to nicosulfuron/rimsulfuron Canadian J. Plant Sci., v. 41, n. 1, p. 151-154, 1997. 